PAPER

\title{
Intravascular lymphoma: magnetic resonance imaging correlates of disease dynamics within the central nervous system
}

\author{
J M Baehring, C Henchcliffe, C J Ledezma, R Fulbright, F H Hochberg
}

J Neurol Neurosurg Psychiatry 2005;76:540-544. doi: 10.1136/jnnp.2003.033662

See end of article for authors' affiliations

.....................

Correspondence to: F H Hochberg,

Massachusetts General

Hospital, Brain Tumor

Center, Cox 315, Boston,

MA 02114, USA:

Fhochberg@partners.org

Received 1 December 2003

In revised form

10 June 2004

Accepted 19 July 2004
Background: Intravascular lymphoma (IVL) is a rare non-Hodgkin's lymphoma with relative predilection for the central nervous system. In the absence of extraneural manifestations, the disease is not recognised until autopsy in the majority of cases underlining the need for new clinical markers.

Methods: This is a retrospective series of five patients with IVL seen at a single institution over three years. An advanced magnetic resonance imaging (MRI) protocol was performed at various time points prior to diagnosis and during treatment.

Results: MRI revealed multiple lesions scattered throughout the cerebral hemispheres; the brainstem, cerebellum, and spinal cord were less frequently involved. On initial presentation, hyperintense lesions were seen on diffusion weighted images suggestive of ischaemia in three of four patients in whom the images were obtained at that time point. In four patients lesions were also identifiable as hyperintense areas on fluid attenuated inversion recovery (FLAIR) sequences. Initial contrast enhancement was encountered in three cases. Diffusion weighted imaging lesions either vanished or followed the typical pattern of an ischaemic small vessel stroke with evolution of abnormal FLAIR signal followed by enhancement with gadolinium in the subacute stage and tissue loss in the chronic stage. Diffusion weighted imaging and FLAIR abnormalities proved to be partially reversible, correlating with the response to chemotherapy.

Conclusion: We provide the first detailed description of the dynamic pattern of diffusion weighted MRI in IVL. These patterns in combination with systemic findings may facilitate early diagnosis and serve as a new tool to monitor treatment response. ntravascular lymphoma (IVL) is a non-Hodgkin's lymphoma, in which the malignant lymphocyte clone is restricted to the lumen of small and medium sized blood vessels. Although a systemic disease, a distinct pattern of organ involvement has been recognised. Nervous system, skin, and parenchymatous organs (adrenal gland) are most commonly involved. Isolated nervous system affliction is frequent and poses the biggest diagnostic challenge since distinction from other microangiopathies such as primary angiitis of the brain is difficult. ${ }^{1}$ Damage to normal tissue likely results from ischaemia caused by small vessel occlusion. Particularly in the absence of extraneural manifestations of IVL, the disease frequently eludes diagnosis since the lesions are small and deep seated and thus prone to sampling error or located in sensitive areas precluding biopsy.

Left untreated, IVL follows a relapsing-remitting or relapsing-progressive clinical course. In order to define radiographic correlates of potential diagnostic benefit, we have retrospectively analysed magnetic resonance imaging (MRI) findings in five cases of IVL. Considering the known ischaemic nature of IVL lesions, we were particularly interested in diffusion weighted MRI (DWI) sequences. Since the introduction of DWI into routine clinical use this issue has not been studied. We also analysed MRI scans obtained after initiation of therapy to investigate if radiographic correlates of treatment response exist.

\section{SUBJECTS AND METHODS}

Between 1999 and 2002, we saw eight patients with IVL on the Neuro-Oncology Service at the Massachusetts General Hospital and Brigham and Women's Hospital. Diagnosis was established by tissue biopsy in three cases (fig 1). In two patients $(72 \mathrm{M}, 79 \mathrm{M})$ in whom a tissue biopsy could not be obtained (critical medical condition in one patient, spinal cord lesions and deep seated, small hemispheric lesions in another patient), spinal fluid analysis in combination with clinical presentation led to the diagnosis of IVL (see table 1). Three patients were not included in this study. In two of these patients we could not establish a morphological, or a flow cytometry based, or a molecular diagnosis of lymphoma. In the third patient, neurological symptoms and radiographic cerebral abnormalities were completely absent.

A standardised advanced magnetic resonance neuroimaging protocol was employed in all patients. In addition to anatomical images prior to and after the administration of gadolinium, physiological information was provided by DWI. A diffusion weighted echo planar imaging sequence was used to obtain data for the generation of apparent diffusion coefficient (ADC) maps. Six high b-value images $\left(1000 \mathrm{~mm}^{2} / \mathrm{s}\right)$ and one low b-value image $\left(3 \mathrm{~mm}^{2} / \mathrm{s}\right)$ were acquired for each of 23 axial slices. A number of excitations of 3 were used for each image to improve the signal to noise ratio. The following parameters were used: $\mathrm{TR}=6 \mathrm{~s}$, $\mathrm{TE}=72.9 \mathrm{~ms}$, diffusion encoding $=47 \mathrm{~ms}, 128 \times 128$ matrix, field of view $22 \times 22,5 \mathrm{~mm}$ slice thickness, $1 \mathrm{~mm}$ spacing. Time points of imaging in this retrospective analysis were not standardised. Frequently, patients underwent multiple MRIs until a diagnosis could be established. Once chemotherapy was initiated, MRI was obtained at least every two months.

Abbreviations: $A D C$, apparent diffusion coefficient; DWI, diffusion weighted MRI; FLAIR, fluid attenuated inversion recovery; IVL, intravascular lymphoma; MRI, magnetic resonance imaging 


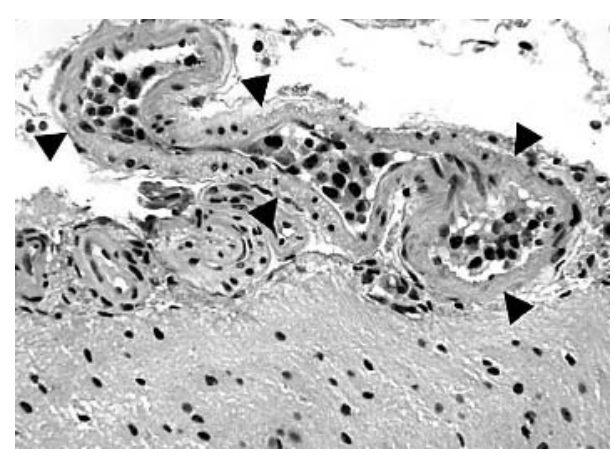

Figure 1 Intravascular lymphoma: malignant lymphocytes fill a leptomeningeal vein (arrowheads; H\&E stain) (case 39F; see table 1).

Three patients were treated with methotrexate chemotherapy $\left(8 \mathrm{~g} / \mathrm{m}^{2}\right)$ in biweekly intervals and one patient with combination chemotherapy (methotrexate, cyclophosphamide, adriamycin, vincristine, prednisone). One patient died prior to initiation of therapy.

\section{RESULTS}

The radiographic data for all five patients included in this study are summarised in table 1 . MRI revealed multiple lesions scattered throughout the cerebral hemispheres as the most common radiographic pattern of IVL observed in all patients. The brainstem (three patients) and cerebellum (one patient) were less frequently involved. Lesions were located deep within the white matter of the hemispheres or close to the cortex (figs 2A, 3A). Two patients were found to have diffuse spinal cord involvement (fig 4) one of whom also had a lumbosacral radiculopathy. One patient presented with optic neuropathy. On initial presentation, usually several days to weeks into the clinical syndrome, hyperintense lesions were seen on diffusion weighted images suggestive of ischaemia in three of four patients in whom these images were obtained at that time point. In another patient DWI showed multiple hemispheric hyperintense lesions 12 days after a normal MRI (fig 2B). In four patients lesions were also identifiable as hyperintense areas on fluid attenuated inversion recovery (FLAIR) sequences. Initial contrast enhancement was encountered in three cases.

DWI lesions either vanished or followed the typical pattern of an ischaemic small vessel stroke with evolution of abnormal FLAIR signal followed by enhancement with gadolinium in the subacute stage and tissue loss in the chronic stage. The subacute to chronic phase of IVL was accompanied by prolongation of $\mathrm{T} 2$ within the area of the lesions likely representing gliosis as the result of chronic ischaemia and direct tissue damage by tumour cells. Cortical lesions ultimately resulted in laminar necrosis (fig 3B).

Untreated IVL followed a recurrent progressive course (fig 3A). Each exacerbation followed the same pattern as described above: new lesions arose, initially characterised by restricted diffusion of protons followed by $\mathrm{T} 2$ prolongation, enhancement, and ultimately tissue loss. At a single time point, lesions at varying stages of evolution were seen.

DWI and FLAIR abnormalities proved to be partially reversible, correlating with the response to chemotherapy (fig 4).

Table 1 Radiographic data of the patients included in the present study

\begin{tabular}{|c|c|c|c|c|}
\hline $\begin{array}{l}\text { Age at } \\
\text { diagnosis, sex }\end{array}$ & $\begin{array}{l}\text { Neurological } \\
\text { symptoms }\end{array}$ & Diagnosis & MRI brain & MRI spine \\
\hline $39 F$ & $\begin{array}{l}\text { Progressive cognitive } \\
\text { decline, headache, } \\
\text { left homonymous } \\
\text { hemianopsia, ataxia }\end{array}$ & $\begin{array}{l}\text { Brain biopsy: intravascular } \\
\text { large B cell lymphoma }\end{array}$ & $\begin{array}{l}\text { Relapsing multifocal lesions involving cortex } \\
\text { and subcortical white matter; enhancement with } \\
\text { gadolinium; acute infarctions (DWI hyperintense) } \\
\text { followed by laminar necrosis and tissue loss }\end{array}$ & Not done \\
\hline $54 \mathrm{~F}$ & $\begin{array}{l}\text { Floaters both eyes, } \\
\text { progressively } \\
\text { diminished vision }\end{array}$ & $\begin{array}{l}\text { Skin biopsy: intravascular } \\
\text { large B cell lymphoma }\end{array}$ & $\begin{array}{l}\text { T2 hyperintense lesion within left optic nerve; } \\
\text { pontine and bilateral subcortical white matter } \\
\text { lesions (FLAIR hyperintense), few of them with } \\
\text { mild restriction of proton diffusion and contrast } \\
\text { enhancement; resolution of DWI, contrast } \\
\text { enhancement and partially of FLAIR } \\
\text { abnormalities with chemotherapy }\end{array}$ & Not done \\
\hline $60 \mathrm{M}$ & Lethargy & $\begin{array}{l}\text { Gastrocnemius muscle } \\
\text { biopsy: intravascular } \\
\text { large B cell lymphoma }\end{array}$ & $\begin{array}{l}\text { Initial scan negative; multiple, mostly cortical } \\
\text { lesions (hyperintense on DWI), evolution over } \\
12 \text { days; no contrast enhancement }\end{array}$ & Not done \\
\hline $72 M$ & $\begin{array}{l}\text { Progressive } \\
\text { paraparesis over } \\
2 \text { weeks, } \\
\text { Korsakoff-like } \\
\text { psychosis }\end{array}$ & $\begin{array}{l}\text { No biopsy done } \\
\text { Cerebrospinal fluid } \\
\text { cytopathology: increased } \\
\text { number of lymphocytes, } \\
\text { some atypical forms } \\
\text { Flow cytometry: CD19+, } \\
\text { CD20+, CD5+, CD10-, } \\
\text { CD23- B cells with excess } \\
\text { expression of } ~ \text { light chain } \\
\text { suggestive of B cell } \\
\text { lymphoma } \\
\text { Clonal lgH gene } \\
\text { rearrangement in spinal } \\
\text { fluid }\end{array}$ & $\begin{array}{l}\text { Heterogeneously enhancing extra-axial frontal } \\
\text { mass; punctate periventricular enhancement; } \\
\text { resolution of contrast enhancement with therapy, } \\
\text { haemorrhage into frontal extra-axial lesion }\end{array}$ & $\begin{array}{l}\text { Diffuse cervicothoracic myelopathy } \\
\text { (hyperintense on FLAIR), punctate } \\
\text { parenchymal and leptomeningeal } \\
\text { enhancement; resolution with } \\
\text { chemotherapy }\end{array}$ \\
\hline $79 M$ & $\begin{array}{l}\text { Progressive } \\
\text { quadriparesis, lower } \\
\text { extremity numbness } \\
\text { over } 1 \text { month, } \\
\text { neurogenic bladder } \\
\text { dysfunction, } \\
\text { encephalopathy, } \\
\text { history of large B } \\
\text { cell lymphoma }\end{array}$ & $\begin{array}{l}\text { Clonal lgH gene } \\
\text { rearrangement in spinal } \\
\text { fluid }\end{array}$ & $\begin{array}{l}\text { Multifocal subcortical small vessel infarcts } \\
\text { (DWI hyperintense) }\end{array}$ & $\begin{array}{l}\text { Hyperintense FLAIR signal and } \\
\text { enhancement, most prominent in } \\
\text { thoracic cord; enlargement, } \\
\text { enhancement of lumbosacral nerve } \\
\text { roots }\end{array}$ \\
\hline
\end{tabular}

DWI, diffusion weighted imaging; FLAIR, fluid attenuated inversion recovery; MRI, magnetic resonance imaging. 

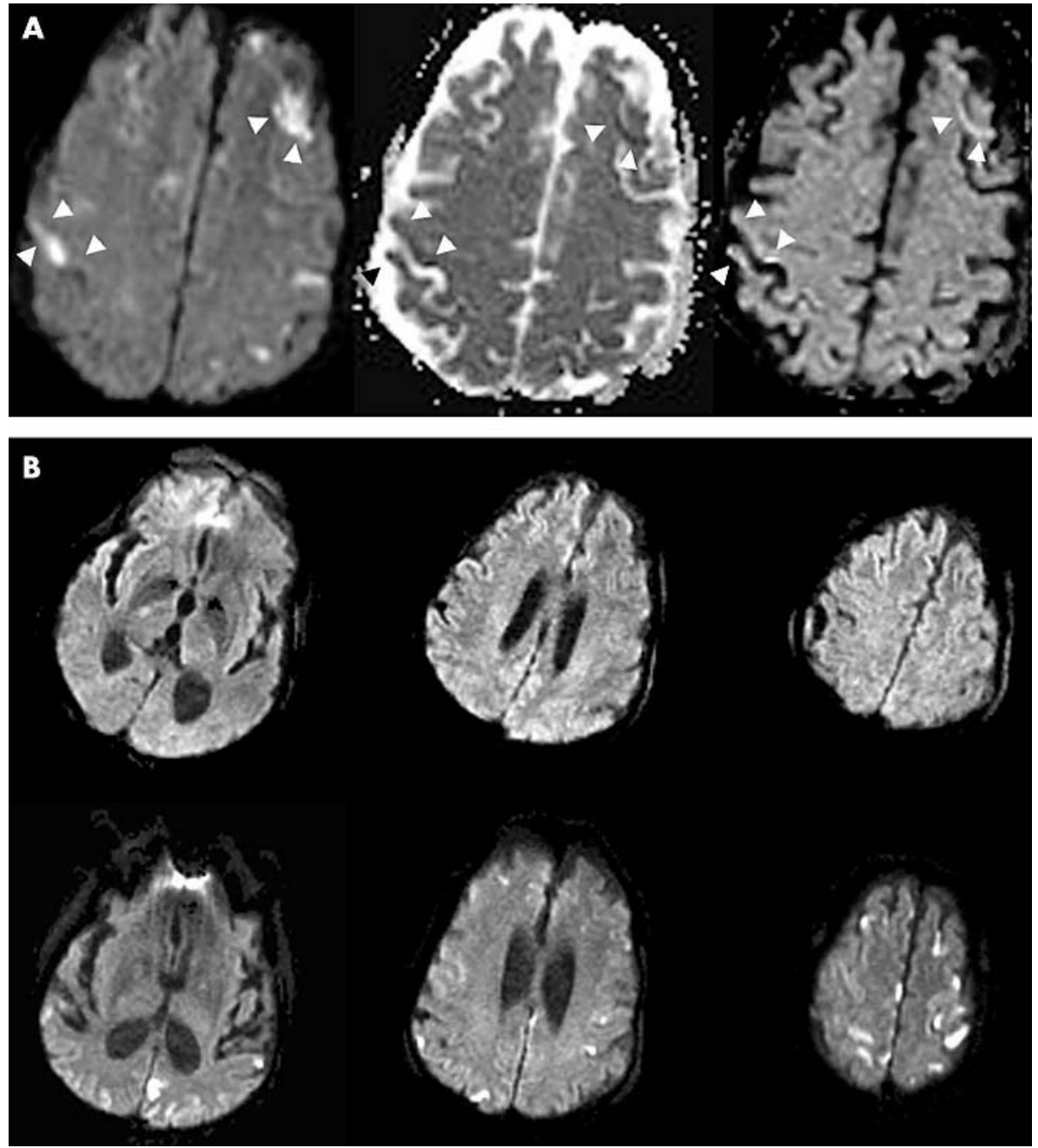

Figure 2 Case 60M. (A) Typical appearance of the acute stage of intravascular lymphoma. Diffusion weighted image (left) shows cortical areas of restricted proton diffusion scattered throughout both hemispheres. At least the left anterior and the right posterior frontal lesions can be identified on the apparent diffusion coefficient map as hypointense signal (middle) and on the exponential image as hyperintense signal. This constellation is consistent with areas of acute ischaemia. (B) The first set of diffusion weighted images (upper three images) was taken at onset of the patient's neurological syndrome (subacute encephalopathy). The second set is from a study 12 days later (lower three images).

\section{DISCUSSION}

We have documented the dynamic evolution and resolution of nervous system lesions in five patients with IVL using MRI.

DWI proved to be the most sensitive MR sequence during the acute phase of IVL. Correlation with extent of disease as estimated by the clinical examination was inconsistent. DWI provides image contrast dependent on the molecular motion of water and has proved most useful in the diagnosis of acute ischaemia. Profound restriction of water diffusion appears within minutes to hours of ischaemia, reflecting possible shifts of water from extracellular to intracellular space or alterations of the sodium/potassium ATP dependent pump mechanism of the cell membrane. ${ }^{2}$ On diffusion weighted images, this results in hyperintense and on ADC maps in hypointense signal. The T2 changes of ischaemia occur somewhat later at 12 hours and gadolinium enhancement within 48 hours. Normalisation of ADC occurs within one to four weeks after the ischaemic event. ${ }^{2}$ DWI changes have also been seen by us and others in the setting of lymphoma masses within brain parenchyma-reflecting possibly the density of lymphoid tumour aggregates. ${ }^{2}{ }^{3}$ Thus DWI changes may accompany both the IVL associated ischaemia and the movement of tumour cells through the vessel wall to the cerebral white matter. Although our data provide evidence that ischaemia represents an important pathogenetic mechanism in IVL, we have observed normal DWI in a symptomatic patient with IVL indicating that other mechanisms exist. In the chronic stage of the disease, FLAIR images served as the best marker for extent of disease. Again, correlation with the clinical picture was frequently poor and its predictive value for outcome was low. Two of our patients had extensive T2 hyperintense signal abnormalities that at least partially resolved with chemotherapy.

As a marker of response to therapy, gadolinium enhancement was less useful than in parenchymal CNS lymphoma. Absence of contrast enhancing lesions does not specifically identify complete remission. We used a combination of clinical variables (resolution of neurological signs and symptoms or extraneural manifestations such as skin rash or autoimmune haemolytic anaemia), DWI data (resolution of lesions, absence of new lesion formation) and gadolinium enhanced MR images to guide chemotherapy. Repeat biopsies of extraneural sites and spinal fluid analyses served as markers of residual disease in selected cases.

Although the usefulness of MRI in IVL with involvement of the nervous system has been pointed out, DWI data have rarely been provided in prior reports of neuroimaging changes in patients with IVL. ${ }^{4-6}$ The most commonly reported MRI findings in IVL are multifocal lesions hyperintense on T2-weighted images. ${ }^{1}{ }^{5}$ - $7-16$ Subcortical white matter is more often affected than cortex, subcortical nuclei, or brainstem structures. Small vessels (arterioles, capillaries, postcapillary venules) are more commonly involved than large vessels 


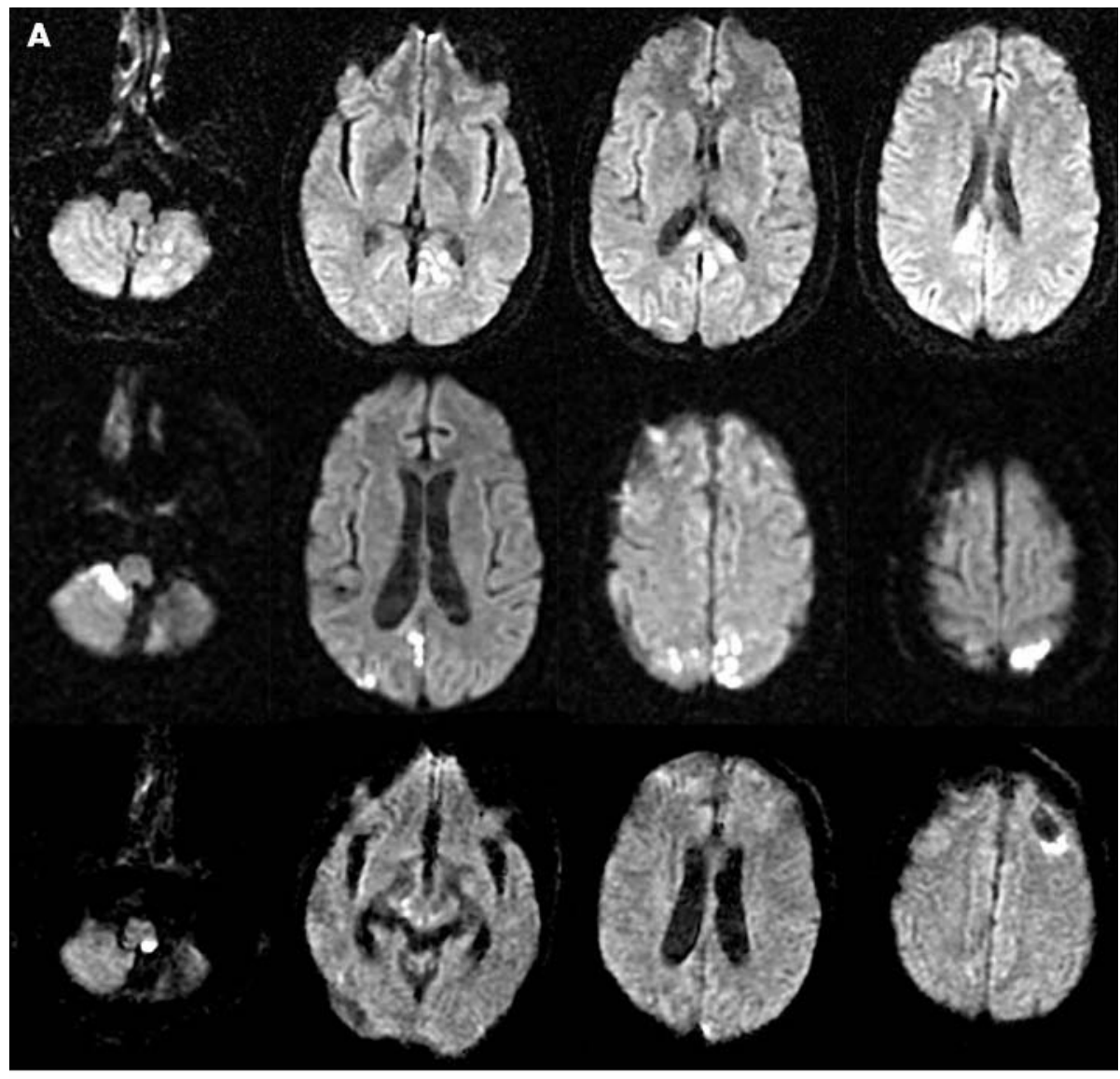

Figure 3 Case 39F. (A) Dynamic evolution and resolution of lesions in a patient with IVL prior to initiation of chemotherapy. Diffusion weighted images are shown from the initial presentation (top row) as well as follow up studies seven months (middle row) and nine months (bottom row) later. Scattered punctate hyperintense areas indicate restricted diffusion of protons consistent with acute ischaemia in

varying vascular territories. (B) Evolution of lesions prior to initiation of chemotherapy from the subacute stage (left: FLAIR sequence showing several areas of infarction involving cortex and subcortical white matter; middle: T1 with gadolinium, gyral pattern of enhancement) to chronic stage (right:

T1 without gadolinium, laminar necrosis, one year later).

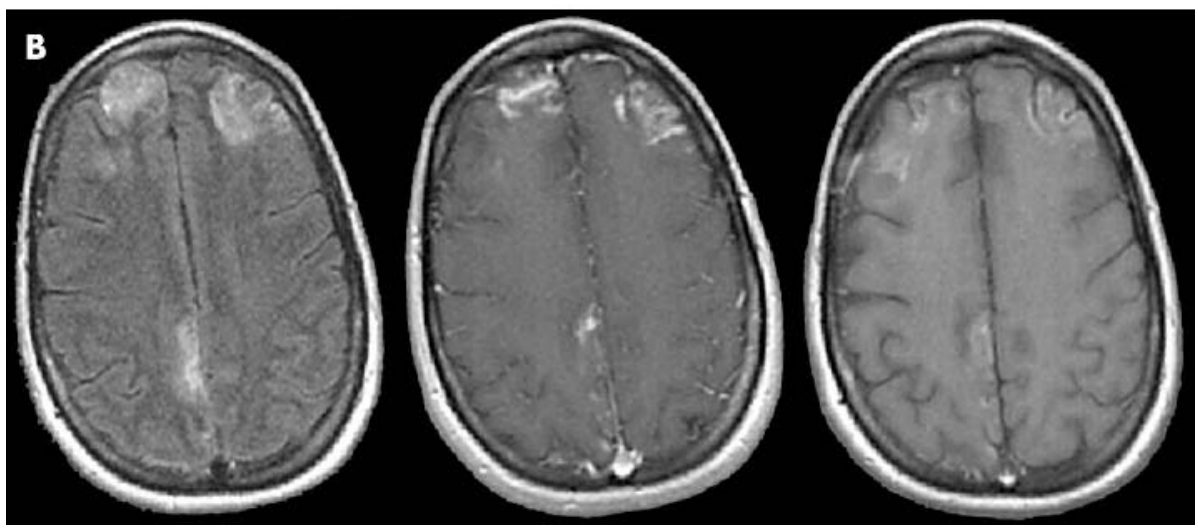

(circle of Willis, dural sinuses; reference 13). IVL can also present as single or multiple mass lesions. ${ }^{12}$ Gadolinium enhancement is dependent upon timing of MRI. In less than a third of cases reported in the literature enhancement was seen on the initial MR scan. Follow up scan revealed enhancement in another 10\%. Dural and/or leptomeningeal involvement as indicated by gadolinium enhancement was present in a small number of patients, ${ }^{4}{ }^{11}{ }^{17}$ as were foci of haemorrhage. ${ }^{16}$ Few cases with normal cerebral MRI scans at initial evaluation were reported either with or without neurological deficits. ${ }^{16}$ Asymptomatic MR lesions appear to be rare (our case; reference 10) but this number is likely underestimated since complete staging evaluations including imaging of the whole neuraxis are not widely performed. Spinal cord involvement is common but sensitivity of MRI is low. Less than $40 \%$ of reported patients with autopsy proven IVL of the cord had an abnormal MRI, invariably T2 hyperintense lesions. ${ }^{101317}$ Few authors have described the dynamic evolution and resolution of contrast enhancing and T2 hyperintense lesions. ${ }^{4} 51016$

The differential diagnosis of IVL and CNS microangiopathies of other aetiologies such as vasculitis remains difficult. ${ }^{4}{ }^{14}$ IVL and primary angiitis of the CNS show a marked overlap of clinical presentation, laboratory findings, and radiographic appearance. Both conditions cause small vessel infarctions scattered throughout the deep white matter of the hemispheres or the spinal cord. ${ }^{5}$ MRI alone cannot solve this diagnostic dilemma.

In two cases of the present series the diagnosis of CNS lymphoma was established but histopathological demonstration of intravascular tumour cells was absent. As an estimated $40-80 \%$ of IVL cases remain undiagnosed until autopsy, these two cases reflect the common clinical situation. Their dynamic pattern of MRI changes did not 


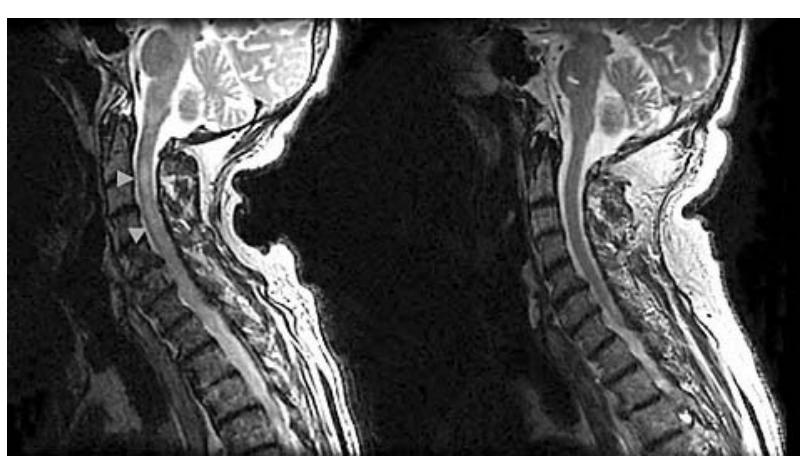

Figure 4 Case 72M. T2-weighted images of the cervical and upper thoracic spine of a patient with intravascular lymphoma at initial presentation (left) and after achievement of partial remission (right; 13 months of high dose methotrexate chemotherapy). Diffuse hyperintense signal abnormality accompanied the patient's clinical syndrome of progressive paraparesis and neurogenic bladder dysfunction. At the time of the second MRI, signal abnormalities had resolved and the patient was walking without assistance.

differ from those observed in the three patients with histopathological diagnosis. By including them in our study, we encourage considering the diagnosis of IVL based upon clinical findings, radiographic appearance, and laboratory studies even if serial biopsies are non-diagnostic or cannot be performed.

Based on our experience and that of others, radiographic IVL diagnosis is suggested by:

(1) multifocal DWI lesions in association with T2 signal abnormalities, confirming the diagnosis of small vessel ischaemia or infarction

(2) gadolinium enhancement appearing in proximity to the T2 or DWI changes, and persisting or enlarging over weeks to months

(3) a dynamic pattern of MRI lesions with resolution of some DWI or T2 lesions and the new appearance of others.

These findings should lead to biopsy of skin, adrenal gland, liver, kidney, brain, or other suspected sites of involvement. The prognosis for IVL has been dismal with mortality over $80 \% .{ }^{1}$ However, chemotherapy may induce a lasting response in patients. ${ }^{15} 18$ Successful therapy depends upon early diagnosis prior to irreversible ischaemic damage and can abolish diffusion, FLAIR abnormalities as well as enhancing foci of tumour accompanying both clinical neurological improvement and resolution of laboratory abnormalities.

\section{Authors' affiliations}

J M Baehring, C J Ledezma, F H Hochberg, Department of Neurology, Massachusetts General Hospital, Harvard Medical School, Boston, MA, USA

C Henchcliffe, Department of Neurology, Columbia Presbyterian Medical Center, New York, NY, USA

J M Baehring, Departments of Neurology and Neurosurgery, Yale University School of Medicine, New Haven, CT, USA (new affiliation) R Fulbright, Department of Diagnostic Radiology, Yale University School of Medicine, New Haven, CT, USA

Competing interests: none declared

\section{REFERENCES}

1 Glass J, Hochberg FH, Miller DC. Intravascular lymphomatosis. A systemic disease with neurologic manifestations. Cancer 1993;71:3156-64.

2 Schaefer PW, Grant PE, Gonzalez RG. Diffusion-weighted MR imaging of the brain. Radiology 2000;217:331-45.

3 Moritani T, Shrier DA, Numaguchi Y, et al. Diffusion-weighted echo-planar MR imaging: clinical applications and piffalls - a pictorial essay. Clin lmaging 2000;24:181-92

4 Calamia KT, Miller A, Shuster EA, et al. Intravascular lymphomatosis. A report of ten patients with central nervous system involvement and a review of the disease process. Adv Exp Med Biol 1999;455:249-65.

5 Liow K, Asmar $\mathrm{P}$, Liow $M$, et al. Intravascular lymphomatosis: contribution of cerebral MRI findings to diagnosis. I Neuroimaging 2000; 10:116-18

6 De Bruecker Y, Demaerel P, Wilms G, et al. A case of hemiparesis associated with cranial nerve lesions due to intravascular lymphomatosis(2003:4b). Eur Radiol 2003;13:1753-5.

7 Demirer T, Dail DH, Aboulafia DM. Four varied cases of intravascular lymphomatosis and a literature review. Cancer 1994;73:1738-45.

8 Liszka U, Drlicek M, Hitzenberger P, et al. Intravascular lymphomatosis: a clinicopathological study of three cases. J Cancer Res Clin Oncol 1994; 120:164-8

9 Sienknecht CW, Whetsell WO, Pollock P. Intravascular malignant lymphoma ("malignant angioendotheliomatosis") mimicking primary angiitis of the central nervous system. J Rheumatol 1995;22:1769-70.

10 Hashimoto $\mathbf{H}$, Naritomi H, Kazui S, et al. Presymptomatic brain lesions on MRI in a patient with intravascular malignant lymphomatosis. J Neuroimaging 1998;8:110-13.

11 Williams RL, Meltzer CC, Smirniotopoulos JG, et al. Cerebral MR imaging in intravascular lymphomatosis. AJNR Am J Neuroradiol 1998; 19:427-31.

12 Massimino M, Giardini R, Cefalo G, et al. Intravascular lymphomatosis (IL) in a child mimicking a posterior fossa tumor. J Neurooncol 2001;51:47-50.

13 Amagasaki K, Yamazaki H, Ohmori K, et al. Malignant intravascular lymphomatosis associated with venous stenosis. Case report. J Neurosurg 1999:90:355-8.

14 al Chalabi A, Sivakumaran M, Holton J, et al. A case of intravascular malignant lymphomatosis (angiotropic lymphoma) with raised perinuclear antineutrophil cytoplasmic antibody titres - a hitherto unreported association. Clin Lab Haematol 1994;16:363-9.

15 DiGiuseppe JA, Nelson WG, Seifter EJ, et al. Intravascular lymphomatosis: a clinicopathologic study of 10 cases and assessment of response to chemotherapy. J Clin Oncol 1994;12:2573-9.

16 Kenez J, Barsi P, Majtenyi K, et al. Can intravascular lymphomatosis mimic sinus thrombosis? A case report with 8 months' follow-up and fatal outcome. Neuroradiology 2000;42:436-40.

17 Martin-Duverneuil N, Mokhtari K, Behin A, et al. Intravascular malignant lymphomatosis. Neuroradiology 2002;44:749-54.

18 Baehring JM, Longtine J, Hochberg FH. A new approach to the diagnosis and treatment of intravascular lymphoma. J Neurooncol 2003;61:237-48. 